\title{
La población y tasas vitales de las misiones jesuíticas de Chiquitos (Bolivia)
}

The population and vital rates of the jesuit missions of Chiquitos (Bolivia)

Robert H. Jackson *

Resumen: Este es el primero de tres artículos que presenta información sobre la población y tasas vitales de las misiones jesuíticas de la provincia de Paraguay reconstruido de censos.

Palabras claves: Chiquitos (Bolivia), tasas vitales, población

Abstract: This is the first of a series of three articles that present information on the population and vital rates of the Jesuit missions in the Paraguay province reconstructed from censuses.

Key words: Chiquitos (Bolivia); vital rates; population

Recibido: 9 de agosto de 2016

Evaluado: 18 de octubre de 2016

*Investigador independiente, Ciudad de México. E-mail: robertvianey@gmail.com

179 Robert H. Jackson. La población y tasas vitales de las misiones jesuíticas.... 179-198. 
La base del estudio de los patrones demográficos de las poblaciones históricas es la reconstrucción de las tasas vitales, de las tasas de natalidad y de mortalidad. En 2015, publiqué un estudio demográfico de las misiones jesuitas de Paraguay y Chiquitos en ingles, titulado: Demographic Change and Ethnic Survival among the Sedentary Populations on the Jesuit Mission Frontiers of South America: The Formation and Persistence of Mission Communities in a Comparative Context.. El libro reconstruyó las tasas vitales de estas comunidades misioneras utilizando censos y los registros bautismos y entierros existentes. El propósito de estos artículos es poner esta información a disposición de un público más amplio en español.

Este artículo no ofrece un análisis detallado de los patrones demográficos de las poblaciones indígenas traídas a vivir en las misiones de Chiquitos. Baste decir que las poblaciones de las misiones de Chiquitos fueron de alta mortalidad y alta fertilidad, y en la mayoría de los años, las tasas de natalidad fueron más altas que las tasas de mortalidad. Además, eran poblaciones abiertas, lo que significa que los jesuitas reasentaron a los no cristianos en las misiones. Esto tiende a inflar el cálculo de las tasas brutas de natalidad en los años en que los jesuitas reasentaron a los no cristianos, pero esto no ocurrió en la mayoría de los años y hay registros de cuándo y cuántos no cristianos fueron llevados a las misiones. En los años con información incompleta, es decir, cuando la población al final del año anterior no está disponible, he estimado la población base restando los bautismos y añadiendo entierros de la población del año actual. Los ensayos han demostrado que este método da resultados razonables cerca de las cifras calculadas con datos completos.

Las fuentes demográficas históricas podrían tener deficiencias, como subenumeraciones porque la gente no tenía dinero para pagar a un sacerdote para administrar sacramentos o porque un censo estaba preparado para propósitos de impuestos o para identificar reclutas militares o para trabajar. Incluso los censos moderados registran subenumeraciones. Los censos misioneros jesuíticos y los registros sacramentales, por otro lado, son generalmente más confiables. Ninguna fuente demográfica es absolutamente exacta, pero los jesuitas no cobraron honorarios sacramentales, y los misioneros usaron fondos comunales para pagar la tribulación. Ciertamente había casos de fugitivos que salían de las misiones, pero en general los residentes de la misión no tenían las mismas motivaciones para evitar aparecer en el registro como lo hicieron, por ejemplo, las poblaciones europeas contemporáneas.

Por otra parte, el análisis de los detallados censos de tributo muestra que los jesuitas pudieron seguir la pista de la población y sabían qué individuos estaban ausentes como fugitivos y por cuánto tiempo, o estaban realizando tareas lejos de las comunidades de la misión. Las epidemias se extendieron a las misiones de Chiquitos. Sin embargo, debido al aislamiento geográfico de los brotes de la región de Chiquitos no alcanzó los niveles catastróficos como en las misiones de Paraguay.

El análisis de los patrones demográficos de las misiones jesuíticas no puede juzgarse con normas basadas en las poblaciones europeas contemporáneas, que eran muy diferentes. Las poblaciones europeas se enfrentaron a restricciones económicas a la formación de la familia que las poblaciones de la misión no lo hicieron. Por ejemplo, la tierra era limitada en Europa, mientras que la tierra era abundante en las misiones y el acceso a la tierra no era un factor que retrasara la formación de la familia en las misiones. Las parejas europeas se casaron a una edad más tardía que en las misiones y las mujeres indígenas se casaron poco después de la pubertad alrededor de los 13 o 14 años. 
La edad de fertilidad potencial era mucho más larga entre las mujeres que vivían en las misiones, lo que dio lugar a fuertes tasas de crecimiento y altas tasas De la fertilidad generalmente más alto que las poblaciones europeas contemporáneas.

Las poblaciones misioneras en Chiquitos crecieron a través de la reproducción natural, y con el reasentamiento periódico de no cristianos. Para una discusión más detallada de los patrones demográficos en las misiones jesuitas de Chiquitos y entre los Guaraní, remito al lector a una serie de mis publicaciones sobre este tema. Ver Jackson (2004, 2005, 2007, 2008, 2014, 2015B, 2015B). Otros estudios sobre las poblaciones de las misiones incluyen las de Maeder y Bolsi (1976, 1978, 1980, 1982), y el artículo de Massimo Livi-Bacci y Maeder (2004), entre otros estudios.

Una serie de gráficos demográficos muestran la trayectoria del cambio de las poblaciones en el tiempo, y en el caso de las misiones Chiquitos ejemplos de la política jesuita de establecer nuevas comunidades mediante la transferencia de población de las misiones existentes. La población de San Rafael, por ejemplo, cayó tras la transferencia de familias para establecer la misión de San Ignacio. Las tablas contienen la siguiente información: población, número de familias y número total de bautizos y entierros. Esta información se ha utilizado para reconstruir las tasas brutas de natalidad y mortalidad por mil habitantes, que es una medida estándar que permite comparar las tasas vitales en el tiempo y entre las comunidades. En otras palabras, el número de bautismos y entierros si la población era de 1.000 personas. Esto se calcula dividiendo 1.000 por la población del año anterior, y luego multiplicando el número de bautismos o entierros. Durante algunos años las tasas vitales se estiman porque la información es incompleta. Esto se hace mediante la estimación de la población del año anterior por la resta de los bautismos y la adición de entierros a la población actual. La cifra final es el tamaño promedio de la familia calculado dividiendo la población total por el número de familias. Mi esperanza es que esta información sea útil para futuras investigaciones.

\begin{tabular}{|c|c|c|c|c|c|c|c|c|c|c|c|c|}
\hline \multicolumn{13}{|c|}{$\begin{array}{l}\text { Catalogo de la Numeracion de las Nifsiones } \\
\text { de chiquitos del Año de } 1764\end{array}$} \\
\hline Pueblos F & Famil!s & Viudos & Virudas $^{\mathbb{N}}$ & $\begin{array}{l}\text { Mucha } \\
\text { chos }\end{array}$ & $\mid \begin{array}{l}\text { Mucha } \\
\text { chas }\end{array}$ & $\begin{array}{l}\text { Baut. } \\
\text { Parv. }\end{array}$ & Baut. & Cafatm & \begin{tabular}{|l|} 
Difurnt \\
adult.
\end{tabular} & \begin{tabular}{|l|} 
Dif. \\
parv.
\end{tabular} & Comur. & Almas \\
\hline S.Xav. ${ }^{r}$ & 703 & Is & 22 & 965 & 848 & 176 & 00 & 33 & 24 & 89 & 2731 & 3256 \\
\hline Concep. $n$ & 684 & 32 & 33 & 974 & 775 & 129 & 00 & So & 52 & 79 & 3418 & 3182 \\
\hline S.Mig: & 267 & 03 & I3 & 401 & 384 & 045 & 00 & 17 & 14 & 26 & 1922 & 1335 \\
\hline S.Ign. ${ }^{\circ}$ & 528 & 04 & 35 & 716 & 749 & 158 & 00 & 26 & $2 I$ & 87 & 3447 & 2560 \\
\hline S.Raf. ${ }^{1}$ & 565 & 14 & 09 & 766 & 713 & 128 & 00 & 34 & 19 & 42 & 2865 & 2632 \\
\hline S. Ana & 350 & 04 & 34 & $46 s$ & 490 & 110 & 00 & $\circ 9$ & Is & 40 & 1967 & 1693 \\
\hline S.Ios. & 513 & 02 & 34 & 621 & 528 & 127 & 00 & I2 & 43 & IOI & 3ISI & $221 I$ \\
\hline S. Inan & 4Is & 17 & $2 I$ & 483 & 463 & 093 & OI & 39 & 23 & 35 & 2796 & 1814 \\
\hline Santiago & 397 & 03 & so & 330 & 348 & 111 & 43 & 24 & 43 & 85 & 24 Is & 1525 \\
\hline S5.coraz? & 567 & os & 49 & 582 & 622 & 108 & 00 & 24 & 12 & 66 & 3200 & 2392 \\
\hline Suma & 4989 & 99 & 300 & 6303 & 5920 & II8S & 44 & 268 & 266 & 650 & $? 7912$ & 22600 \\
\hline
\end{tabular}






Los censos generales de las misiones de Chiquitos en 1764.
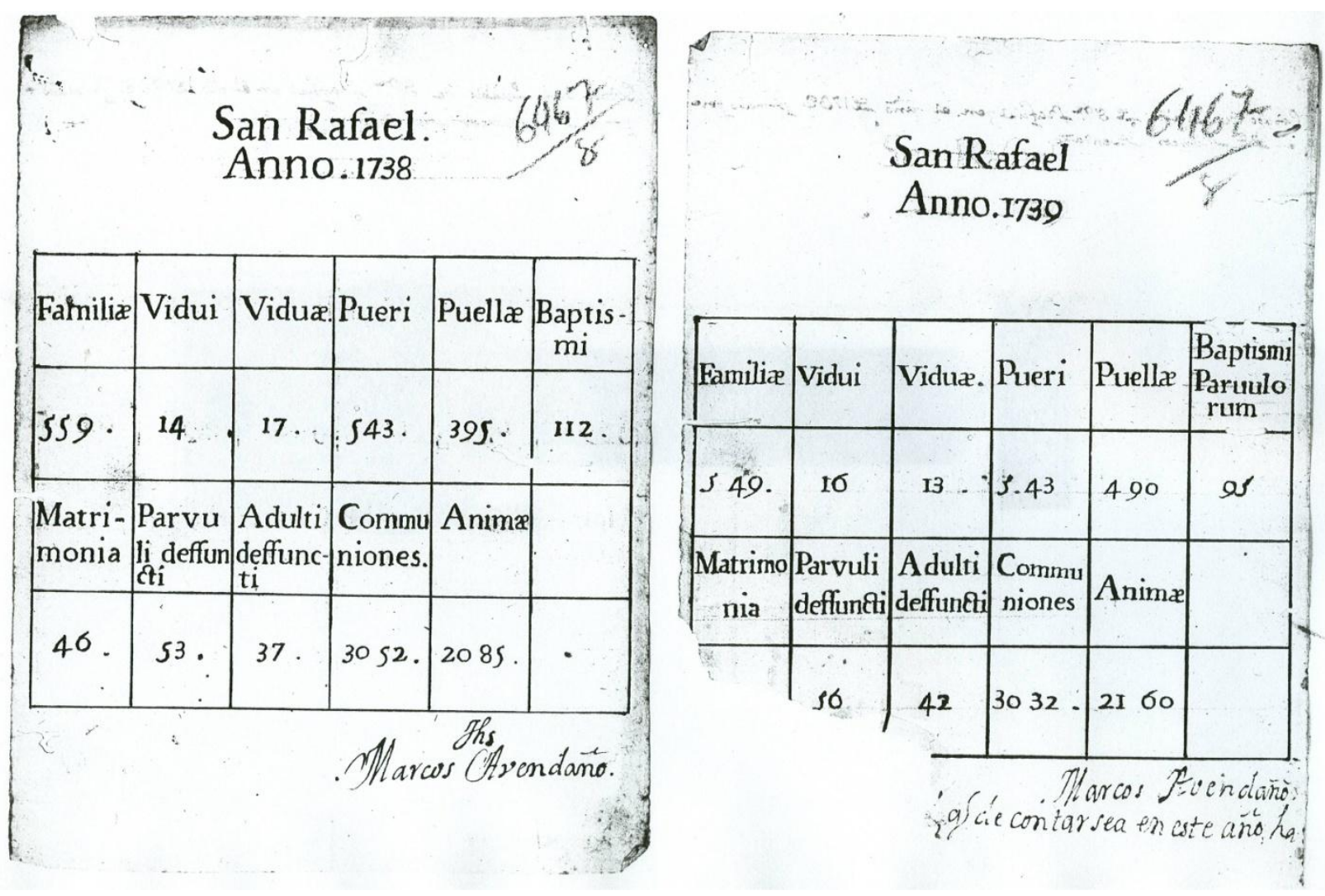

Los censos de San Rafael en 1738 y 1739. 


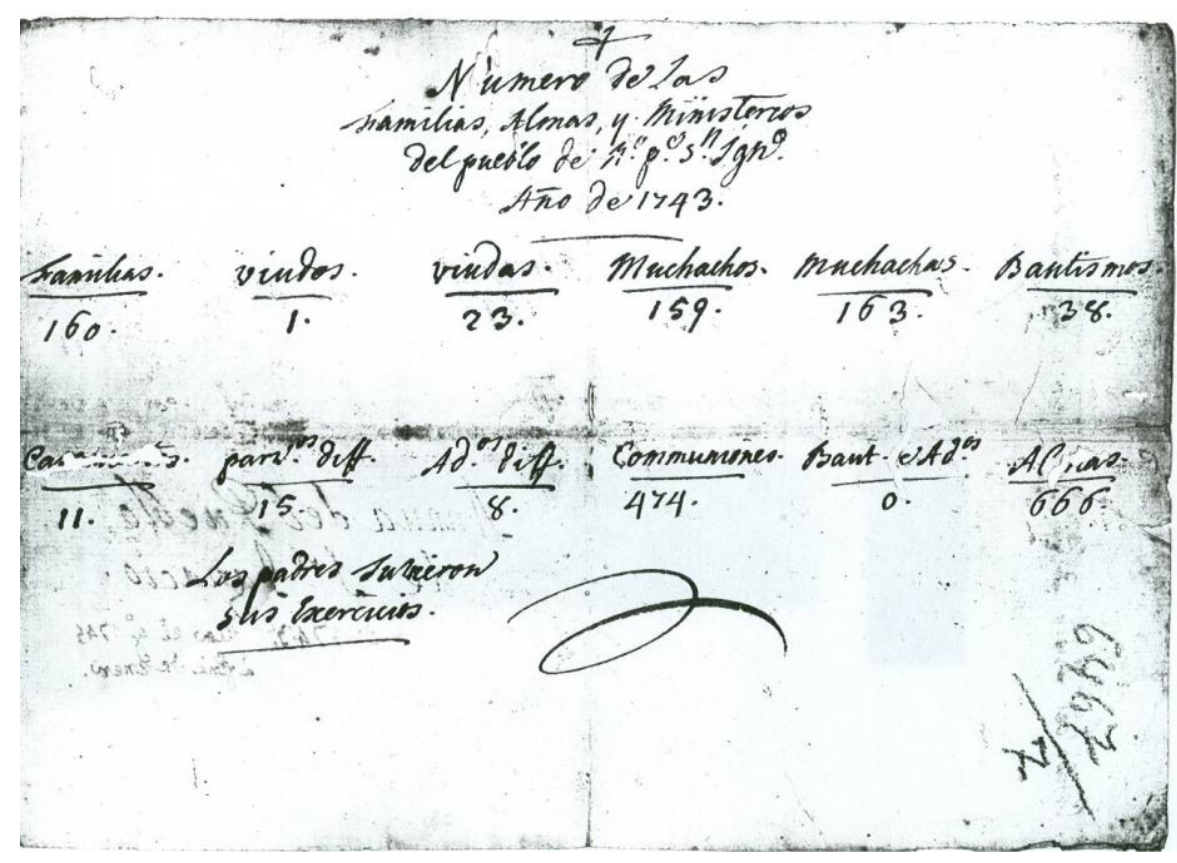

El censo de 1743 de San Ignacio.

La Población y tasas vitales de San Francisco Xavier, 1712-1768

\begin{tabular}{|l|c|c|c|c|c|c|c|c|}
\hline \multicolumn{2}{|c|}{} & \multicolumn{2}{|c|}{ Bautismos } & \multicolumn{2}{l|}{} \\
\hline Año & Families & Población & Niños & Adultos & Entierros & $\begin{array}{c}\text { Tasa } \\
\text { Bruta de } \\
\text { Fertilidad }\end{array}$ & $\begin{array}{c}\text { Tasa Bruta } \\
\text { de } \\
\text { Mortalidad }\end{array}$ & TPF* \\
\hline 1712 & 478 & 1955 & 93 & & 19 & $49.4^{*}$ & $10.1^{*}$ & 4.0 \\
\hline 1718 & 505 & 1688 & 75 & & 22 & 44.6 & 13.1 & 3.3 \\
\hline 1735 & 605 & 2345 & 109 & & 94 & $46.8 *$ & $40.3^{*}$ & 3.9 \\
\hline 1738 & 559 & 2342 & 138 & 7 & 189 & $57.8 *$ & $79.1 *$ & 4.2 \\
\hline 1739 & 560 & 2364 & 111 & 1 & 191 & 47.4 & 81.6 & 4.2 \\
\hline 1740 & 564 & 2481 & 120 & 2 & 65 & 50.8 & 27.5 & 4.4 \\
\hline 1741 & 558 & 2378 & 130 & 3 & 75 & 52.4 & 30.2 & 4.3 \\
\hline 1742 & 545 & 2413 & 135 & 3 & 110 & 56.8 & 46.3 & 4.4 \\
\hline 1743 & 546 & 2416 & 131 & & 138 & 55.1 & 58.0 & 4.4 \\
\hline 1744 & 556 & 2403 & 127 & & 140 & 52.6 & 58.0 & 4.3 \\
\hline 1745 & 552 & 2293 & 115 & & 138 & 47.9 & 57.4 & 4.2 \\
\hline 1746 & 582 & 2314 & 125 & & 71 & 54.5 & 31.0 & 4.0 \\
\hline 1747 & 612 & 2435 & 115 & & 144 & 49.7 & 62.2 & 4.0 \\
\hline 1748 & 620 & 2497 & 153 & & 91 & 62.8 & 37.4 & 4.0 \\
\hline 1749 & 622 & 2480 & 115 & & 130 & 46.1 & 52.1 & 4.0 \\
\hline 1750 & 633 & 2550 & 156 & & 86 & 62.9 & 34.7 & 4.0 \\
\hline 1751 & & & 72 & & & 28.2 & & \\
\hline
\end{tabular}


IHS. Antiguos jesuitas en Iberoamérica ISSN: 2314-3908

Vol. 5 no 1

enero-junio 2017

\begin{tabular}{|c|c|c|c|c|c|c|c|c|}
\hline 1752 & 568 & 2323 & 72 & & & & & 4.1 \\
\hline 1753 & & & 216 & & & & & \\
\hline 1754 & & & 156 & & & & & \\
\hline 1755 & 606 & 2578 & 158 & & 92 & $62.9^{*}$ & $36.6^{*}$ & 4.3 \\
\hline 1756 & 615 & 2639 & 165 & & 104 & 64.0 & 40.3 & 4.3 \\
\hline 1757 & 631 & 2728 & 156 & & 57 & 59.1 & 21.6 & 4.3 \\
\hline 1758 & 642 & 2799 & 154 & & 83 & 56.5 & 30.4 & 4.4 \\
\hline 1759 & & & 170 & & & & & \\
\hline 1760 & 656 & 2978 & 171 & & 101 & $58.8^{*}$ & $34.7^{*}$ & 4.5 \\
\hline 1761 & 666 & 3065 & 191 & & 104 & 64.1 & 34.9 & 4.6 \\
\hline 1762 & & & 158 & & & 51.6 & & \\
\hline 1763 & & & 194 & & & & & \\
\hline 1764 & 703 & 3256 & 176 & & 113 & $55.1^{*}$ & $35.4^{*}$ & 4.6 \\
\hline 1765 & 728 & 3302 & 198 & & 142 & 60.8 & 43.6 & 4.5 \\
\hline 1766 & 720 & 3201 & 164 & & 265 & 49.7 & 80.3 & 4.5 \\
\hline 1767 & & & 173 & 2 & & & & \\
\hline 1768 & & 2022 & 147 & & & 45.9 & & \\
\hline
\end{tabular}

*Estimada. **TPF - Tamaño Promedio de la Familia.

Fuente: Robert H. Jackson, Demographic Change and Ethnic Survival among the Sedentary Populations on the Jesuit Mission Frontiers of South America: The Formation and Persistence of Mission Communities in a Comparative Context (Leiden: Brill, 2015), 260.

\section{La Población de San Francisco Xavier, 1710-1823}

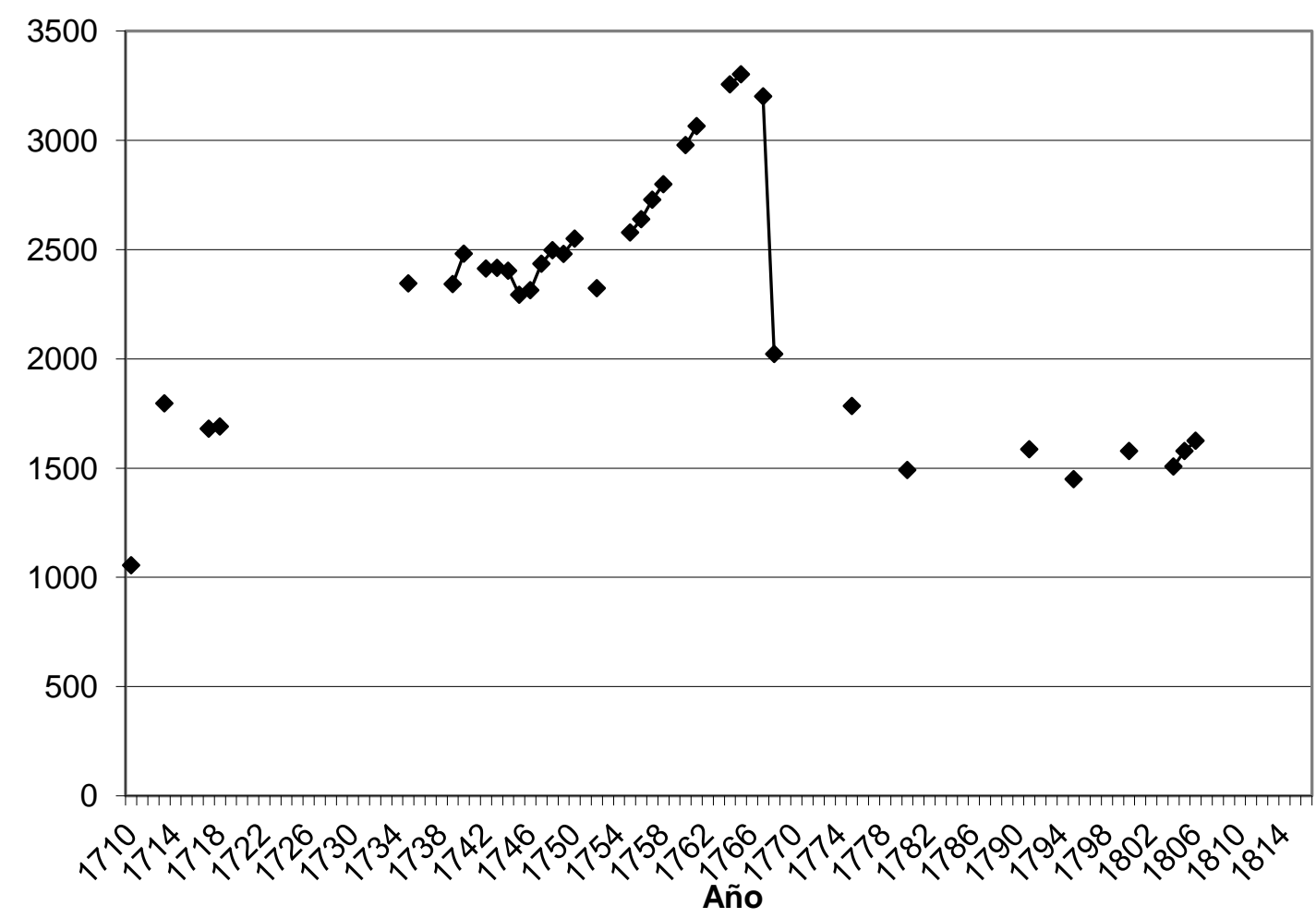

185 Robert H. Jackson. La población y tasas vitales de las misiones jesuíticas.... 179-198. 
La Población y tasas vitales de San Rafael, 1712-1766

\begin{tabular}{|c|c|c|c|c|c|c|c|c|}
\hline \multirow[b]{2}{*}{ Año } & \multirow[b]{2}{*}{ Families } & \multirow[b]{2}{*}{ Población } & \multicolumn{2}{|c|}{ Bautismos } & \multirow[b]{2}{*}{ Entierros } & \multirow[b]{2}{*}{$\begin{array}{c}\text { Tasa } \\
\text { Bruta de } \\
\text { Fertilidad }\end{array}$} & \multirow[b]{2}{*}{$\begin{array}{c}\text { Tasa Bruta } \\
\text { de } \\
\text { Mortalidad }\end{array}$} & \multirow[b]{2}{*}{ TPF** } \\
\hline & & & Niños & Adultos & & & & \\
\hline 1712 & 306 & 1275 & 50 & 25 & 32 & $39.8 *$ & $25.5^{*}$ & 4.2 \\
\hline 1734 & 554 & 2081 & 187 & 48 & 109 & $93.4 *$ & $54.4^{*}$ & 3.8 \\
\hline 1735 & 570 & 2109 & 119 & 22 & 96 & 57.2 & 46.1 & 3.7 \\
\hline 1738 & 559 & 2085 & 112 & 0 & 90 & $54.3^{*}$ & $43.6^{*}$ & 3.7 \\
\hline 1739 & 549 & 2160 & 95 & 0 & 92 & 45.6 & 44.1 & 3.9 \\
\hline 1740 & 546 & 2201 & 80 & 0 & 58 & 37.0 & 26.4 & 4.0 \\
\hline 1741 & 492 & 2075 & 116 & 28 & 52 & 52.7 & 23.6 & 4.2 \\
\hline 1742 & 491 & 2144 & 129 & 0 & 57 & 62.2 & 27.5 & 4.4 \\
\hline 1743 & 494 & 2196 & 127 & 0 & 77 & 61.2 & 37.1 & 4.5 \\
\hline 1744 & 493 & 2256 & 122 & 0 & 100 & 55.6 & 45.5 & 4.6 \\
\hline 1745 & 495 & 2323 & 134 & 0 & 67 & 59.4 & 29.7 & 4.7 \\
\hline 1746 & 510 & 2411 & 146 & 0 & 58 & 62.9 & 25.0 & 4.7 \\
\hline 1747 & 505 & 2497 & 142 & 0 & 56 & 58.9 & 23.2 & 5.0 \\
\hline 1748 & 534 & 2543 & 130 & 0 & 84 & 52.1 & 33.6 & 4.8 \\
\hline 1749 & 559 & 2613 & 129 & 0 & 59 & 50.7 & 23.2 & 4.7 \\
\hline 1750 & 568 & 2749 & 187 & 0 & 51 & 71.6 & 19.5 & 4.8 \\
\hline 1755 & 403 & 1959 & 98 & 0 & 52 & $51.2 *$ & $27.2^{*}$ & 4.9 \\
\hline 1756 & 416 & 2038 & 131 & 0 & 52 & 64.3 & 26.5 & 4.9 \\
\hline 1757 & 440 & 2120 & 100 & 0 & 36 & 49.1 & 17.7 & 4.8 \\
\hline 1758 & 470 & 2173 & 113 & 0 & 42 & 53.3 & 19.8 & 4.6 \\
\hline 1760 & 483 & 2311 & 122 & 0 & 74 & $53.9 *$ & $32.7 *$ & 4.8 \\
\hline 1761 & 509 & 2374 & 121 & 0 & 58 & 52.4 & 25.1 & 4.7 \\
\hline 1764 & 565 & 2632 & 128 & 0 & 61 & $49.9 *$ & $23.8^{*}$ & 4.7 \\
\hline 1765 & 571 & 2733 & 175 & 0 & 74 & 66.5 & 28.1 & 4.8 \\
\hline 1766 & 562 & 2746 & 157 & 0 & 91 & 57.5 & 33.3 & 4.9 \\
\hline
\end{tabular}

*Estimada. **TPF - Tamaño Promedio de la Familia.

Source: Robert H. Jackson, Demographic Change and Ethnic Survival among the Sedentary Populations on the Jesuit Mission Frontiers of South America: The Formation and Persistence of Mission Communities in a Comparative Context (Leiden: Brill, 2015), 265. 
La Población de San Rafael, 1710-1823

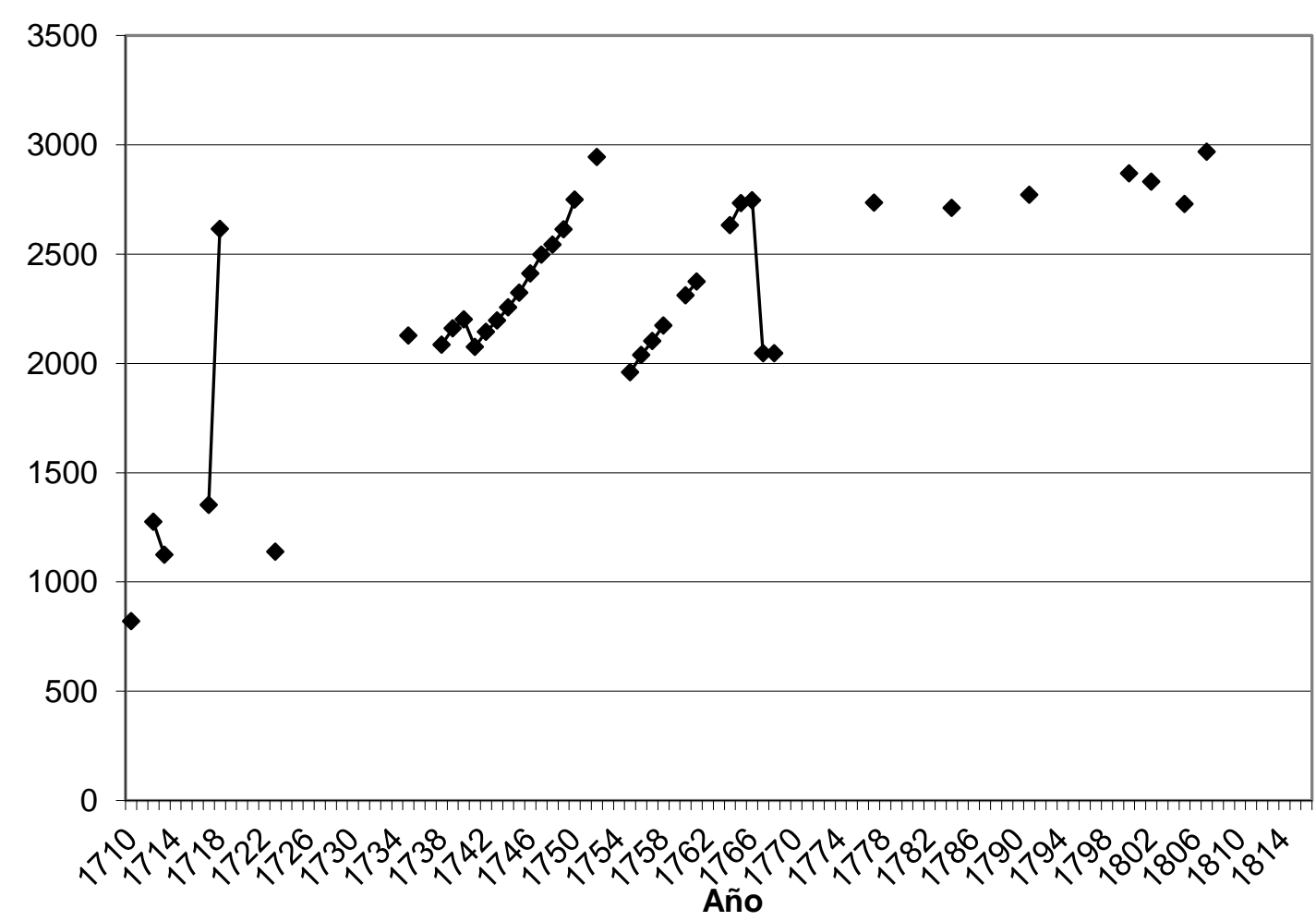

La Población y tasas vitales de San José, 1712-1766

\begin{tabular}{|c|c|c|c|c|c|c|c|c|}
\hline & & & Bat & smos & & & & \\
\hline Año & Families & Población & Niños & Adultos & Entierros & $\begin{array}{c}\text { Tasa } \\
\text { Bruta de } \\
\text { Fertilidad }\end{array}$ & $\begin{array}{c}\text { Tasa Bruta } \\
\text { de } \\
\text { Mortalidad }\end{array}$ & TPF** \\
\hline 1712 & 507 & 1275 & 31 & 0 & N/A & $24.9 *$ & N/A & 2.5 \\
\hline 1734 & 423 & 1832 & 143 & 0 & 62 & $81.7^{*}$ & $35.4^{*}$ & 4.3 \\
\hline 1735 & 426 & 1911 & 128 & 0 & 60 & 69.9 & 32.8 & 4.5 \\
\hline 1738 & 436 & 2011 & 140 & 0 & 64 & $72.4^{*}$ & $33.1^{*}$ & 4.6 \\
\hline 1740 & 455 & 2195 & 153 & 0 & 59 & $76.1 *$ & $29.3 *$ & 4.6 \\
\hline 1742 & 505 & 2409 & 164 & 0 & 63 & $71.0^{*}$ & $27.3 *$ & 4.8 \\
\hline 1743 & 512 & 2439 & 145 & 0 & 93 & 62.9 & 40.3 & 4.8 \\
\hline 1744 & 472 & 2218 & 118 & 0 & 265 & 48.4 & 108.7 & 4.7 \\
\hline 1745 & 505 & 2477 & 177 & 0 & 101 & 79.8 & 45.5 & 4.0 \\
\hline 1746 & 610 & 2916 & 178 & 0 & 156 & 71.9 & 63.0 & 4.8 \\
\hline 1747 & 615 & 2879 & 176 & 0 & 155 & 61.1 & 53.2 & 4.7 \\
\hline
\end{tabular}


IHS. Antiguos jesuitas en Iberoamérica ISSN: 2314-3908

Vol. 5 no 1

enero-junio 2017

\begin{tabular}{|l|c|c|c|c|c|c|c|c|}
\hline 1748 & 614 & 2803 & 169 & 0 & 131 & 58.7 & 45.5 & 4.9 \\
\hline 1749 & 628 & 2783 & 113 & 0 & 142 & 40.3 & 50.7 & 4.4 \\
\hline 1750 & 630 & 2831 & 178 & 0 & 130 & 64.0 & 46.7 & 4.5 \\
\hline 1755 & 563 & 2428 & 120 & 0 & 169 & $48.5^{*}$ & $68.2^{*}$ & 4.3 \\
\hline 1756 & 455 & 2024 & 156 & 0 & 81 & 64.3 & 33.4 & 4.5 \\
\hline 1757 & 455 & 2074 & 122 & 0 & 69 & 60.3 & 34.1 & 4.6 \\
\hline 1758 & 478 & 2139 & 148 & 0 & 84 & 71.4 & 40.5 & 4.5 \\
\hline 1760 & 492 & 2208 & 132 & 0 & 61 & $61.8^{*}$ & $28.6^{*}$ & 4.5 \\
\hline 1761 & 496 & 2186 & 109 & 0 & 132 & 49.4 & 59.8 & 4.4 \\
\hline 1764 & 513 & 2211 & 127 & 0 & 144 & $57.0^{*}$ & $64.6^{*}$ & 4.3 \\
\hline 1765 & 473 & 2242 & 182 & 3 & 168 & 82.3 & 76.0 & 4.7 \\
\hline 1766 & 618 & 2715 & 99 & 0 & 245 & 44.2 & 109.3 & 4.4 \\
\hline
\end{tabular}

*Estimada. **TPF - Tamaño Promedio de la Familia.

Fuente: Robert H. Jackson, Demographic Change and Ethnic Survival among the Sedentary Populations on the Jesuit Mission Frontiers of South America: The Formation and Persistence of Mission Communities in a Comparative Context (Leiden: Brill, 2015), 262.

\section{La Población de San José. 1710-1823}

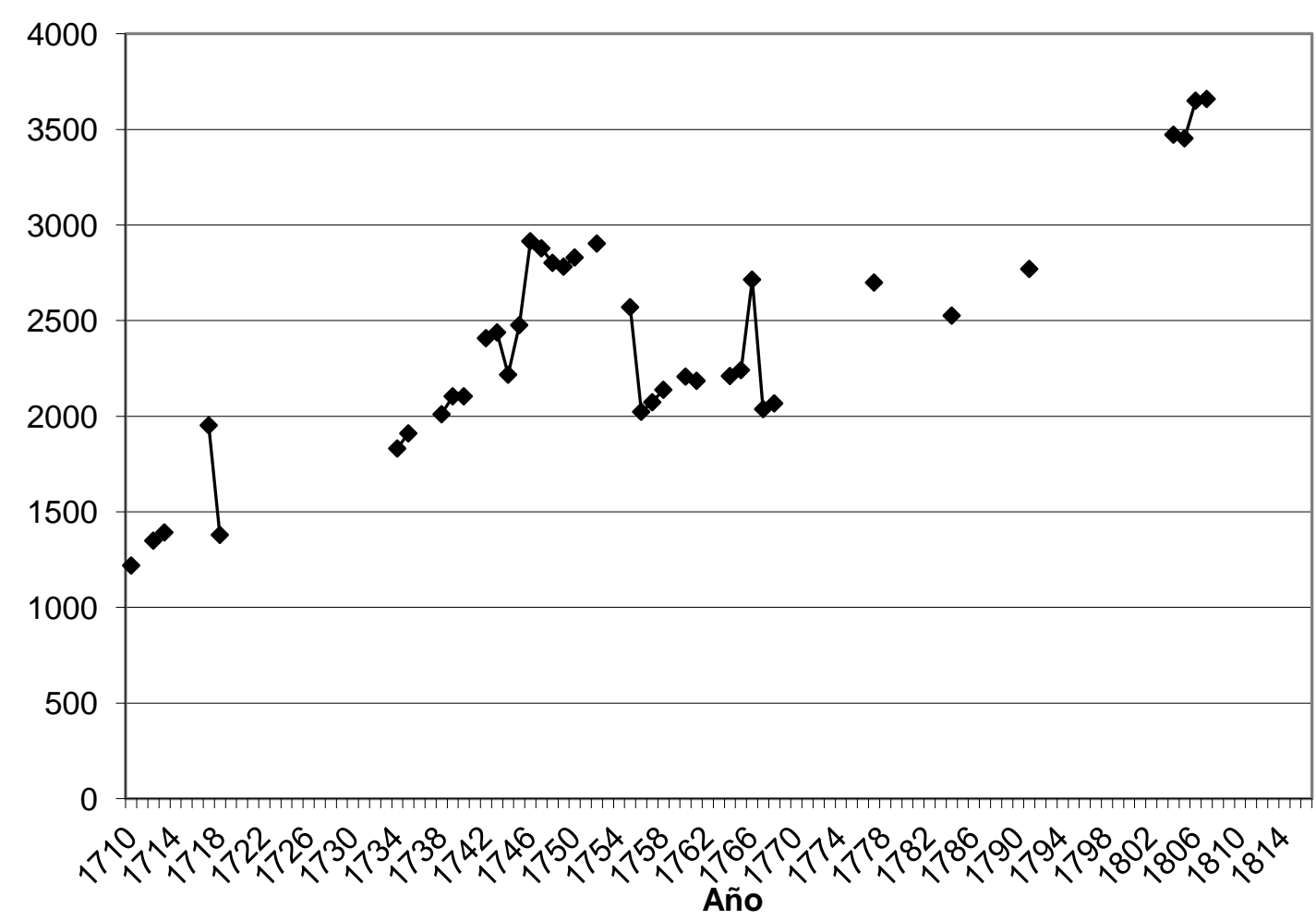

188 Robert H. Jackson. La población y tasas vitales de las misiones jesuíticas.... 179-198. 
La Población y tasas vitales de San Juan Bautista, 1734-1766

\begin{tabular}{|c|c|c|c|c|c|c|c|c|}
\hline \multirow[b]{2}{*}{ Año } & \multirow[b]{2}{*}{ Families } & \multirow[b]{2}{*}{ Población } & \multicolumn{2}{|c|}{ Bautismos } & \multirow[b]{2}{*}{ Entierros } & \multirow[b]{2}{*}{$\begin{array}{c}\text { Tasa } \\
\text { Bruta de } \\
\text { Fertilidad }\end{array}$} & \multirow[b]{2}{*}{$\begin{array}{c}\text { Tasa Bruta } \\
\text { de } \\
\text { Mortalidad }\end{array}$} & \multirow[b]{2}{*}{ TPF** } \\
\hline & & & Niños & Adultos & & & & \\
\hline 1734 & 428 & 1992 & 116 & 16 & 66 & $59.7 *$ & $34.0 *$ & 4.7 \\
\hline 1736 & 382 & 1615 & 92 & 0 & 76 & 46.2 & 38.2 & 4.2 \\
\hline 1738 & 420 & 1793 & 94 & 0 & 40 & $54.1 *$ & $23.0 *$ & 4.2 \\
\hline 1739 & 428 & 1839 & 96 & 0 & 58 & 53.5 & 32.4 & 4.3 \\
\hline 1740 & 428 & 1839 & 96 & 2 & 58 & 52.2 & 31.5 & 4.3 \\
\hline 1741 & 410 & 1820 & 94 & 0 & 61 & 51.1 & 33.2 & 4.4 \\
\hline 1742 & 400 & 1927 & 85 & 0 & 67 & 46.7 & 36.8 & 4.8 \\
\hline 1743 & 398 & 1970 & 92 & 0 & 64 & 50.6 & 35.2 & 5.0 \\
\hline 1744 & 387 & 1855 & 83 & 0 & 183 & 42.1 & 92.9 & 4.8 \\
\hline 1745 & 384 & 1981 & 100 & 0 & 62 & 53.9 & 33.4 & 5.2 \\
\hline 1746 & 430 & 2097 & 116 & 0 & 150 & 83.8 & 75.7 & 4.9 \\
\hline 1747 & 466 & 2091 & 111 & 0 & 76 & 52.9 & 36.2 & 4.5 \\
\hline 1748 & 439 & 1880 & 99 & 0 & 84 & 47.4 & 40.2 & 4.3 \\
\hline 1749 & 412 & 1737 & 50 & 0 & 222 & 26.6 & 118.1 & 4.2 \\
\hline 1750 & 409 & 1726 & 80 & 0 & 91 & 46.1 & 52.4 & 4.2 \\
\hline 1755 & 433 & 1855 & 95 & 7 & 64 & $52.1 *$ & $35.1 *$ & 4.3 \\
\hline 1756 & 447 & 1811 & 143 & 6 & 75 & 77.1 & 40.4 & 4.1 \\
\hline 1757 & 471 & 1918 & 139 & 59 & 44 & 76.8 & 24.3 & 4.1 \\
\hline 1758 & 464 & 2272 & 87 & 8 & 83 & 45.4 & 43.3 & 4.9 \\
\hline 1760 & 479 & 2049 & 113 & 6 & 65 & $56.5^{*}$ & $28.6^{*}$ & 4.3 \\
\hline 1761 & 451 & 2006 & 94 & 4 & 62 & 45.9 & 30.3 & 4.4 \\
\hline 1764 & 415 & 1814 & 93 & 1 & 58 & $52.3^{*}$ & $32.6^{*}$ & 4.4 \\
\hline 1765 & 418 & 1883 & 119 & 3 & 52 & 85.6 & 28.7 & 4.5 \\
\hline 1766 & 425 & 1953 & 116 & 0 & 46 & 61.6 & 24.4 & 4.6 \\
\hline
\end{tabular}

*Estimada. **TPF - Tamaño Promedio de la Familia.

Fuente: Robert H. Jackson, Demographic Change and Ethnic Survival among the Sedentary Populations on the Jesuit Mission Frontiers of South America: The Formation and Persistence of Mission Communities in a Comparative Context (Leiden: Brill, 2015), 263. 


\section{La Población de San Juan Bautista. 1710-1823}

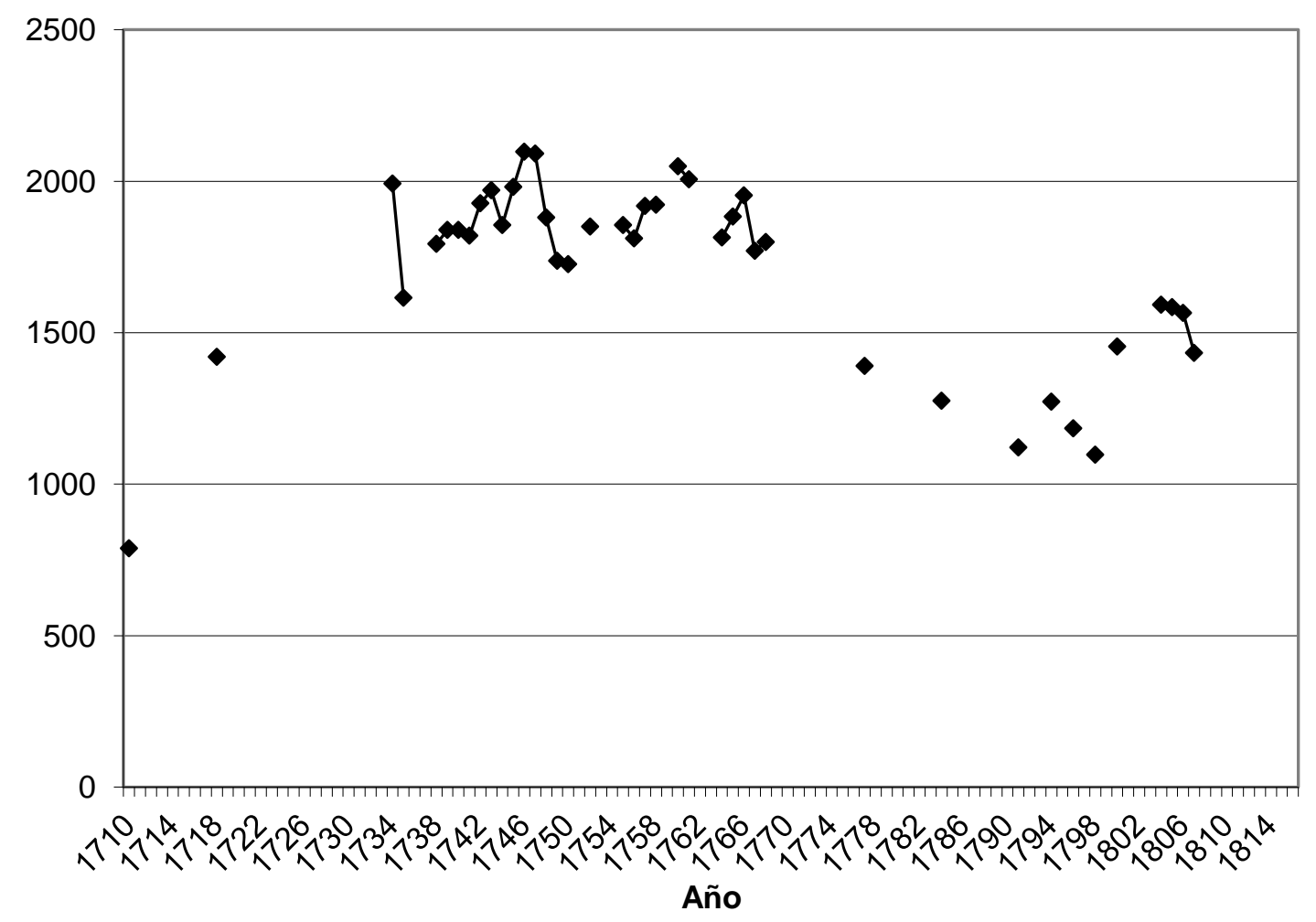

La Población y tasas vitales de las tres misiones nombradas San Ignacio, 1710-1776

\begin{tabular}{|c|c|c|c|c|c|c|c|c|}
\hline \multirow{2}{*}{ Año } & \multirow[b]{2}{*}{ Families } & \multirow[b]{2}{*}{ Población } & \multicolumn{2}{|c|}{ Bautismos } & \multirow[b]{2}{*}{ Entierros } & \multirow[b]{2}{*}{$\begin{array}{c}\text { Tasa } \\
\text { Bruta de } \\
\text { Fertilidad }\end{array}$} & \multirow[b]{2}{*}{$\begin{array}{c}\text { Tasa Bruta } \\
\text { de } \\
\text { Mortalidad }\end{array}$} & \multirow[b]{2}{*}{ TPF** } \\
\hline & & & Niños & Adultos & & & & \\
\hline \multicolumn{9}{|c|}{ San Ignacio de Boacocas (establecida 1707) } \\
\hline 1710 & 94 & 330 & & & & & & 3.5 \\
\hline \multicolumn{9}{|c|}{ San Ignacio de Zamucos (establecida 1723) } \\
\hline 1738 & 144 & 587 & 37 & 7 & 19 & $65.0 *$ & $33.4 *$ & 4.1 \\
\hline 1739 & 149 & 635 & 40 & 0 & 16 & 68.1 & 27.3 & 4.3 \\
\hline 1740 & 156 & 634 & 13 & 0 & 11 & 20.5 & 17.3 & 4.1 \\
\hline 1741 & 159 & 649 & 35 & 2 & 27 & 55.2 & 42.6 & 4.1 \\
\hline 1742 & 156 & 648 & 42 & 0 & 23 & 64.7 & 35.4 & 4.2 \\
\hline 1743 & 160 & 666 & 38 & 0 & 23 & 58.6 & 35.4 & 4.2 \\
\hline 1744 & 165 & 679 & 29 & 0 & 21 & 43.5 & 31.5 & 4.1 \\
\hline \multicolumn{9}{|c|}{ San Ignacio de Chiquitos (establecida 1748) } \\
\hline 1748 & 390 & 1694 & 39 & 0 & 6 & $23.5^{*}$ & $3.6^{*}$ & 4.3 \\
\hline
\end{tabular}


IHS. Antiguos jesuitas en Iberoamérica ISSN: 2314-3908

Vol. 5 no 1

enero-junio 2017

\begin{tabular}{|c|c|c|c|c|c|c|c|c|}
\hline 1749 & 356 & 1624 & 60 & 9 & 30 & 37.0 & 17.7 & 4.6 \\
\hline 1750 & 374 & 1682 & 81 & 0 & 23 & 49.9 & 14.2 & 4.5 \\
\hline 1755 & 425 & 1990 & 125 & 0 & 36 & $65.8^{*}$ & $18.9^{*}$ & 4.7 \\
\hline 1756 & 435 & 2106 & 137 & 0 & 49 & 68.8 & 24.6 & 4.8 \\
\hline 1757 & 443 & 2196 & 133 & 0 & 52 & 63.2 & 24.7 & 5.0 \\
\hline 1758 & 466 & 2144 & 137 & 0 & 46 & 62.4 & 21.0 & 4.6 \\
\hline 1760 & 483 & 2339 & 144 & 0 & 82 & $63.2 *$ & $36.0 *$ & 4.8 \\
\hline 1761 & 510 & 2382 & 149 & 0 & 70 & 63.7 & 29.9 & 4.7 \\
\hline 1764 & 528 & 2560 & 158 & 0 & 108 & $63.0 *$ & $43.0 *$ & 4.9 \\
\hline 1765 & 520 & 2645 & 167 & 0 & 82 & 65.2 & 32.0 & 5.1 \\
\hline 1766 & 531 & 2734 & 167 & 0 & 78 & 63.1 & 29.5 & 5.2 \\
\hline
\end{tabular}

*Estimada. **TPF - Tamaño Promedio de la Familia.

Fuente: Robert H. Jackson, Demographic Change and Ethnic Survival among the Sedentary Populations on the Jesuit Mission Frontiers of South America: The Formation and Persistence of Mission Communities in a Comparative Context (Leiden: Brill, 2015), 266.

La Población de las tres misiones nombradas San Ignacio (1710, 1718-1744, 1748-1819)



191 Robert H. Jackson. La población y tasas vitales de las misiones jesuíticas.... 179-198. 
IHS. Antiguos jesuitas en Iberoamérica ISSN: 2314-3908

Vol. 5 no 1

enero-junio 2017

La Población y tasas vitales de Concepción, 1734-1766

\begin{tabular}{|c|c|c|c|c|c|c|c|c|}
\hline \multirow[b]{2}{*}{ Año } & \multirow[b]{2}{*}{ Families } & \multirow[b]{2}{*}{ Población } & \multicolumn{2}{|c|}{ Bautismos } & \multirow[b]{2}{*}{ Entierros } & \multirow[b]{2}{*}{$\begin{array}{c}\text { Tasa } \\
\text { Bruta de } \\
\text { Fertilidad }\end{array}$} & \multirow[b]{2}{*}{$\begin{array}{c}\text { Tasa Bruta } \\
\text { de } \\
\text { Mortalidad }\end{array}$} & \multirow[b]{2}{*}{ TPF** } \\
\hline & & & Niños & Adultos & & & & \\
\hline 1734 & 403 & 1672 & 109 & 0 & 49 & $67.6 *$ & $30.4 *$ & 4.2 \\
\hline 1736 & 415 & 1721 & 48 & 0 & 31 & $28.2 *$ & $18.2 *$ & 4.2 \\
\hline 1738 & 435 & 1858 & 85 & 0 & 57 & $46.5^{*}$ & $31.2 *$ & 4.3 \\
\hline 1739 & 436 & 1828 & 77 & 0 & 130 & 41.4 & 70.0 & 4.2 \\
\hline 1740 & 436 & 1828 & 77 & 0 & 90 & 42.1 & 49.2 & 4.2 \\
\hline 1741 & 435 & 1846 & 101 & 0 & 114 & 55.3 & 61.8 & 4.2 \\
\hline 1742 & 451 & 1868 & 78 & 0 & 59 & 42.3 & 32.0 & 4.1 \\
\hline 1743 & 458 & 1912 & 109 & 0 & 64 & 59.1 & 34.7 & 4.2 \\
\hline 1744 & 481 & 1950 & 92 & 0 & 54 & 48.1 & 28.2 & 4.1 \\
\hline 1745 & 496 & 2055 & 107 & 0 & 35 & 54.9 & 18.0 & 4.1 \\
\hline 1746 & 498 & 2131 & 118 & 0 & 42 & 57.4 & 20.4 & 4.3 \\
\hline 1747 & 524 & 2212 & 133 & 0 & 52 & 62.4 & 24.4 & 4.2 \\
\hline 1748 & 525 & 2260 & 132 & 0 & 71 & 59.7 & 32.1 & 4.3 \\
\hline 1749 & 587 & 2556 & 130 & 0 & 48 & 47.7 & 33.7 & 4.4 \\
\hline 1750 & 593 & 2992 & 122 & 0 & 86 & 57.5 & 21.2 & 4.4 \\
\hline 1755 & 600 & 2597 & 135 & 0 & 79 & $53.1 *$ & $31.1 *$ & 4.3 \\
\hline 1756 & 594 & 2703 & 184 & 0 & 78 & 70.9 & 30.0 & 4.6 \\
\hline 1757 & 616 & 2778 & 148 & 0 & 73 & 54.8 & 27.0 & 4.5 \\
\hline 1758 & 624 & 2865 & 167 & 0 & 80 & 60.1 & 28.8 & 4.6 \\
\hline 1760 & 672 & 2978 & 152 & 0 & 98 & $52.0 *$ & $33.5^{*}$ & 4.4 \\
\hline 1761 & 672 & 3039 & 142 & 0 & 93 & 47.7 & 31.2 & 4.5 \\
\hline 1764 & 684 & 3182 & 129 & 0 & 131 & $40.5^{*}$ & $41.1^{*}$ & 4.7 \\
\hline 1765 & 687 & 3287 & 227 & 0 & 94 & 71.3 & 29.5 & 4.8 \\
\hline 1766 & 713 & 3278 & 178 & 0 & 183 & 54.2 & 55.7 & 4.6 \\
\hline
\end{tabular}

*Estimada. **TPF - Tamaño Promedio de la Familia.

Fuente: Robert H. Jackson, Demographic Change and Ethnic Survival among the Sedentary Populations on the Jesuit Mission Frontiers of South America: The Formation and Persistence of Mission Communities in a Comparative Context (Leiden: Brill, 2015), 261. 
La Población de Concepción, 1710-1823

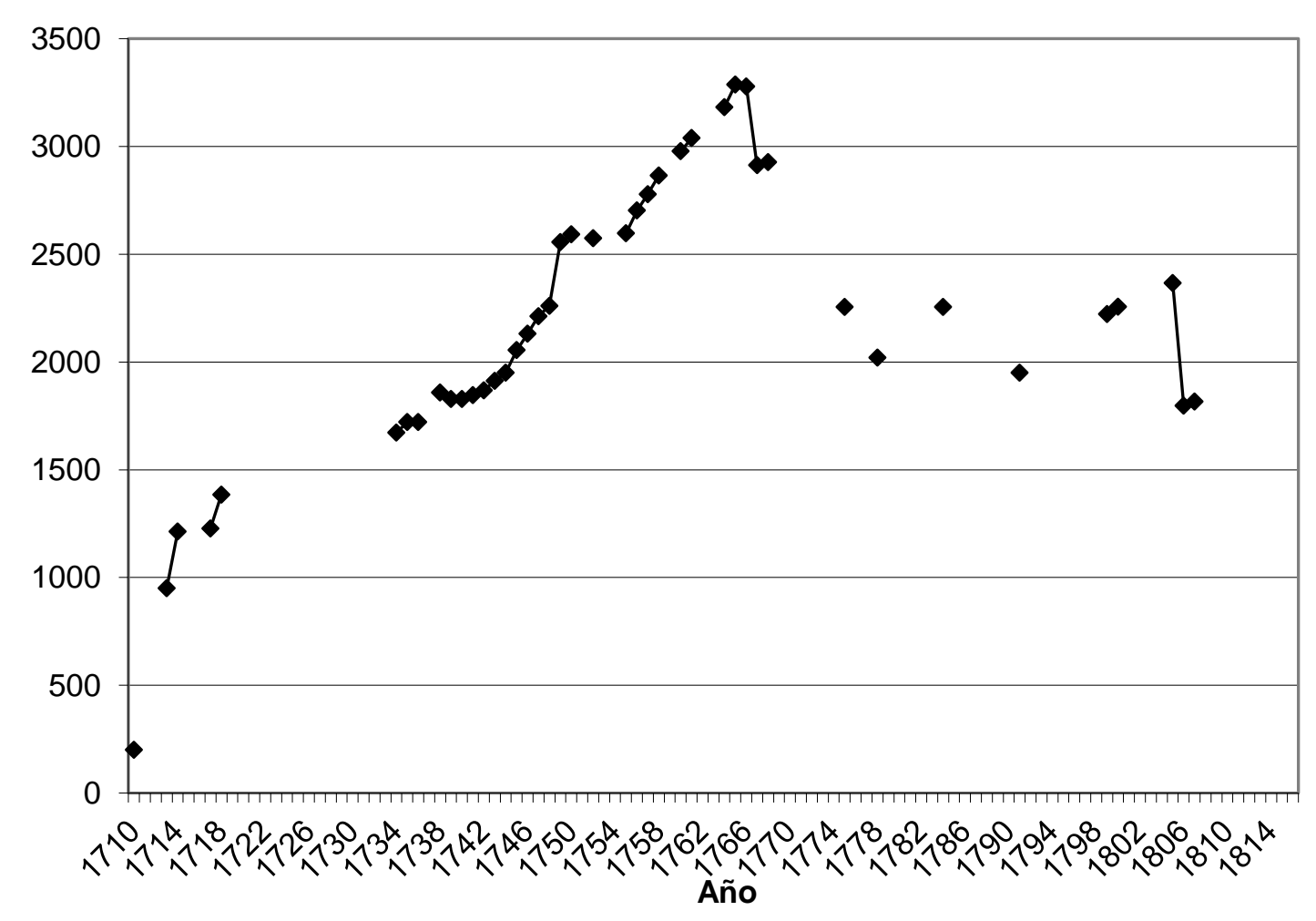

La Población y tasas vitales de San Miguel, 1735-1766

\begin{tabular}{|c|c|c|c|c|c|c|c|c|}
\hline & & & Bat & smos & & & & \\
\hline Año & Families & Población & Niños & Adultos & Entierros & $\begin{array}{c}\text { Tasa } \\
\text { Bruta de } \\
\text { Fertilidad }\end{array}$ & $\begin{array}{c}\text { Tasa Bruta } \\
\text { de } \\
\text { Mortalidad }\end{array}$ & TPF** $^{* *}$ \\
\hline 1735 & 457 & 2242 & 192 & 0 & 132 & $88.0^{*}$ & $60.5^{*}$ & 4. 9 \\
\hline 1738 & 494 & 2208 & 102 & 0 & 66 & $47.0^{*}$ & $30.4 *$ & 4.5 \\
\hline 1739 & 514 & 2283 & 121 & 0 & 47 & 54.8 & 21.3 & 4.4 \\
\hline 1740 & 537 & 2300 & 100 & 0 & 27 & 43.8 & 11.8 & 4.3 \\
\hline 1741 & 535 & 2477 & 118 & 0 & 156 & 22.3 & 67.8 & 4.6 \\
\hline 1742 & 557 & 2580 & 171 & 0 & 69 & 69.0 & 27.9 & 4.6 \\
\hline 1743 & 576 & 2633 & 150 & 30 & 71 & 60.6 & 28.7 & 4.6 \\
\hline 1744 & 657 & 2935 & 170 & 40 & 84 & 64.6 & 31.9 & 4.5 \\
\hline 1745 & 661 & 2955 & 199 & 0 & 56 & 67.8 & 19.1 & 4.5 \\
\hline 1746 & 703 & 3130 & 195 & 0 & 48 & 66.0 & 16.2 & 4.6 \\
\hline 1747 & 732 & 3271 & 193 & 5 & 101 & 61.7 & 32.3 & 4.5 \\
\hline 1748 & 453 & 1972 & 127 & 0 & 46 & 38.8 & 14.1 & 4.4 \\
\hline
\end{tabular}


IHS. Antiguos jesuitas en Iberoamérica ISSN: 2314-3908

Vol. 5 no 1

enero-junio 2017

\begin{tabular}{|c|c|c|c|c|c|c|c|c|}
\hline 1749 & 427 & 1995 & 121 & 0 & 40 & 61.4 & 20.1 & 4.7 \\
\hline 1750 & 458 & 2029 & 102 & 0 & 78 & 51.1 & 39.1 & 4.4 \\
\hline 1755 & 566 & 2500 & 162 & 0 & 71 & $67.2^{*}$ & $29.5^{*}$ & 4.4 \\
\hline 1756 & 600 & 2619 & 167 & 0 & 48 & 66.8 & 19.2 & 4.4 \\
\hline 1757 & 617 & 2689 & 158 & 0 & 88 & 60.3 & 33.6 & 4.4 \\
\hline 1758 & 637 & 2822 & 197 & 0 & 64 & 73.3 & 23.8 & 4.4 \\
\hline 1760 & 668 & 2956 & 190 & 0 & 69 & $67.0 *$ & $24.3 *$ & 4.4 \\
\hline 1761 & 280 & 1219 & 71 & 0 & 42 & 59.7 & 35.3 & 4.4 \\
\hline 1764 & 267 & 1335 & 45 & 0 & 40 & $33.8 *$ & $30.1 *$ & 5.0 \\
\hline 1765 & 280 & 1429 & 115 & 0 & 43 & 86.1 & 32.2 & 5.1 \\
\hline 1766 & 295 & 1473 & 69 & 0 & 183 & 33.8 & 128.1 & 5.0 \\
\hline
\end{tabular}

*Estimada. **TPF - Tamaño Promedio de la Familia.

Fuente: Robert H. Jackson, Demographic Change and Ethnic Survival among the Sedentary Populations on the Jesuit Mission Frontiers of South America: The Formation and Persistence of Mission Communities in a Comparative Context (Leiden: Brill, 2015), 264.

\section{La Población de San Miguel, 1735-1823}

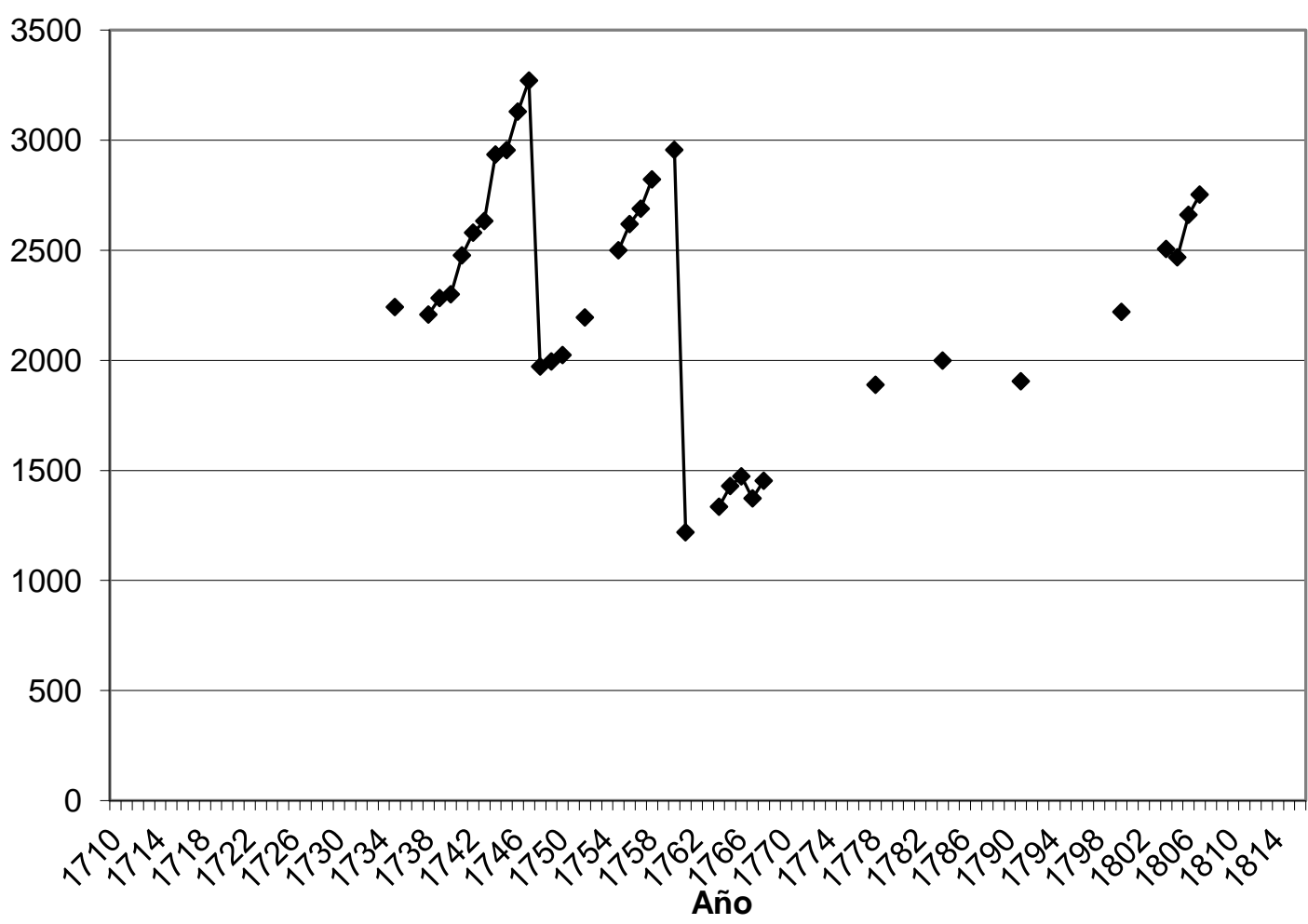

194 Robert H. Jackson. La población y tasas vitales de las misiones jesuíticas.... 179-198. 
La Población y tasas vitales de Santiago, 1755-1766

\begin{tabular}{|l|c|c|c|c|c|c|c|c|}
\hline \multicolumn{2}{|c|}{} & \multicolumn{2}{|c|}{ Bautismos } & \multicolumn{3}{c|}{} \\
\hline Año & Families & Población & Niños & Adultos & Entierros & $\begin{array}{c}\text { Tasa } \\
\text { Bruta de } \\
\text { Fertilidad }\end{array}$ & $\begin{array}{c}\text { Tasa Bruta } \\
\text { de } \\
\text { Mortalidad }\end{array}$ & TPF** \\
\hline 1755 & 237 & 882 & 23 & 0 & 73 & $21.9^{*}$ & $69.5^{*}$ & 3.7 \\
\hline 1756 & 396 & 1460 & 88 & 0 & 42 & 100.0 & 47.6 & 3.7 \\
\hline 1757 & 376 & 1390 & 91 & 0 & 81 & 62.3 & 55.5 & 3.7 \\
\hline 1758 & 379 & 1440 & 116 & 0 & 88 & 83.5 & 63.3 & 3.8 \\
\hline 1760 & 444 & 1718 & 111 & 9 & 101 & $65.0 *$ & $59.1 *$ & 3.9 \\
\hline 1761 & 343 & 1387 & 99 & 18 & 169 & 57.6 & 98.4 & 4.0 \\
\hline 1764 & 397 & 1525 & 111 & 43 & 128 & $72.0 *$ & $83.0 *$ & 3.8 \\
\hline 1765 & 397 & 1556 & 117 & 170 & 133 & 76.7 & 87.2 & 3.9 \\
\hline 1766 & 410 & 1614 & 117 & 0 & 55 & 75.2 & 35.3 & 3.9 \\
\hline
\end{tabular}

*Estimada. **TPF - Tamaño Promedio de la Familia.

Fuente: Robert H. Jackson, Demographic Change and Ethnic Survival among the Sedentary Populations on the Jesuit Mission Frontiers of South America: The Formation and Persistence of Mission Communities in a Comparative Context (Leiden: Brill, 2015), 267.

\section{La Población de Santiago, 1755-1823}




La Población y tasas vitales de Santa Ana, 1755-1766

\begin{tabular}{|l|c|c|c|c|c|c|c|c|}
\hline \multicolumn{2}{|c|}{} & \multicolumn{2}{|c|}{ Bautismos } & \multicolumn{3}{c|}{} \\
\hline Año & Families & Población & Niños & Adultos & Entierros & $\begin{array}{c}\text { Tasa } \\
\text { Bruta de } \\
\text { Fertilidad }\end{array}$ & $\begin{array}{c}\text { Tasa Bruta } \\
\text { de } \\
\text { Mortalidad }\end{array}$ & TPF** \\
\hline 1755 & 254 & 1295 & 42 & 0 & 22 & $32.9^{*}$ & $17.3^{*}$ & 5.1 \\
\hline 1756 & 266 & 1334 & 80 & 0 & 31 & 61.8 & 23.9 & 5.0 \\
\hline 1757 & 281 & 1359 & 67 & 0 & 41 & 50.2 & 30.7 & 4.8 \\
\hline 1758 & 295 & 1412 & 99 & 0 & 49 & 72.9 & 36.1 & 4.8 \\
\hline 1760 & 306 & 1482 & 86 & 0 & 44 & $59.7 *$ & $30.6 *$ & 4.8 \\
\hline 1761 & 310 & 1511 & 89 & 0 & 48 & 60.1 & 32.4 & 4.9 \\
\hline 1764 & 350 & 1693 & 110 & 0 & 55 & $67.2 *$ & $33.6^{*}$ & 4.8 \\
\hline 1765 & 363 & 1771 & 119 & 0 & 45 & 70.3 & 26.6 & 4.9 \\
\hline 1766 & 367 & 1787 & 102 & 0 & 98 & 57.6 & 55.3 & 4.9 \\
\hline
\end{tabular}

*Estimada. **TPF - Tamaño Promedio de la Familia.

Fuente: Robert H. Jackson, Demographic Change and Ethnic Survival among the Sedentary Populations on the Jesuit Mission Frontiers of South America: The Formation and Persistence of Mission Communities in a Comparative Context (Leiden: Brill, 2015), 267.

La Población de Santa Ana, 1755-1823

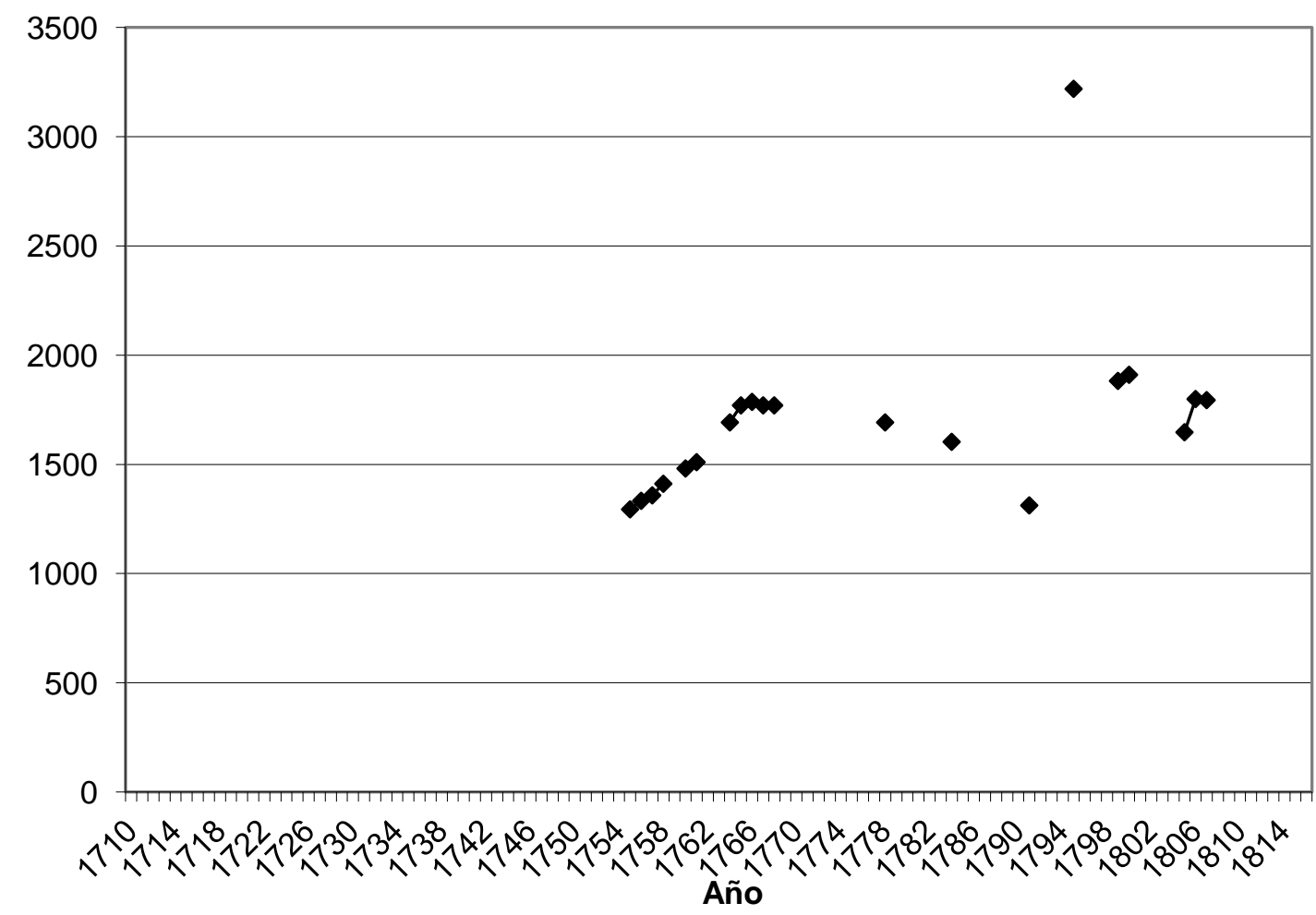


La Población y tasas vitales de Santo Corazón, 1761-1766

\begin{tabular}{|l|c|c|c|c|c|c|c|c|}
\hline \multicolumn{2}{|c|}{} & \multicolumn{2}{|c|}{ Bautismos } & \multicolumn{3}{|c|}{} \\
\hline Año & Families & Población & Niños & Adultos & Entierros & $\begin{array}{c}\text { Tasa } \\
\text { Bruta de } \\
\text { Fertilidad }\end{array}$ & $\begin{array}{c}\text { Tasa Bruta } \\
\text { de } \\
\text { Mortalidad }\end{array}$ & TPF** \\
\hline 1761 & 415 & 1697 & 18 & 0 & 70 & $10.3^{*}$ & $40.0^{*}$ & 4.1 \\
\hline 1764 & 567 & 2392 & 108 & 0 & 88 & $45.6^{*}$ & $37.1^{*}$ & 4.2 \\
\hline 1765 & 544 & 2440 & 203 & 0 & 143 & 84.7 & 59.8 & 4.5 \\
\hline 1766 & 532 & 2287 & 94 & 0 & 219 & 38.5 & 89.8 & 4.3 \\
\hline
\end{tabular}

*Estimada. **TPF - Tamaño Promedio de la Familia.

Fuente: Robert H. Jackson, Demographic Change and Ethnic Survival among the Sedentary Populations on the Jesuit Mission Frontiers of South America: The Formation and Persistence of Mission Communities in a Comparative Context (Leiden: Brill, 2015), 268.

\section{La Población de Santo Corazón, 1761-1823}

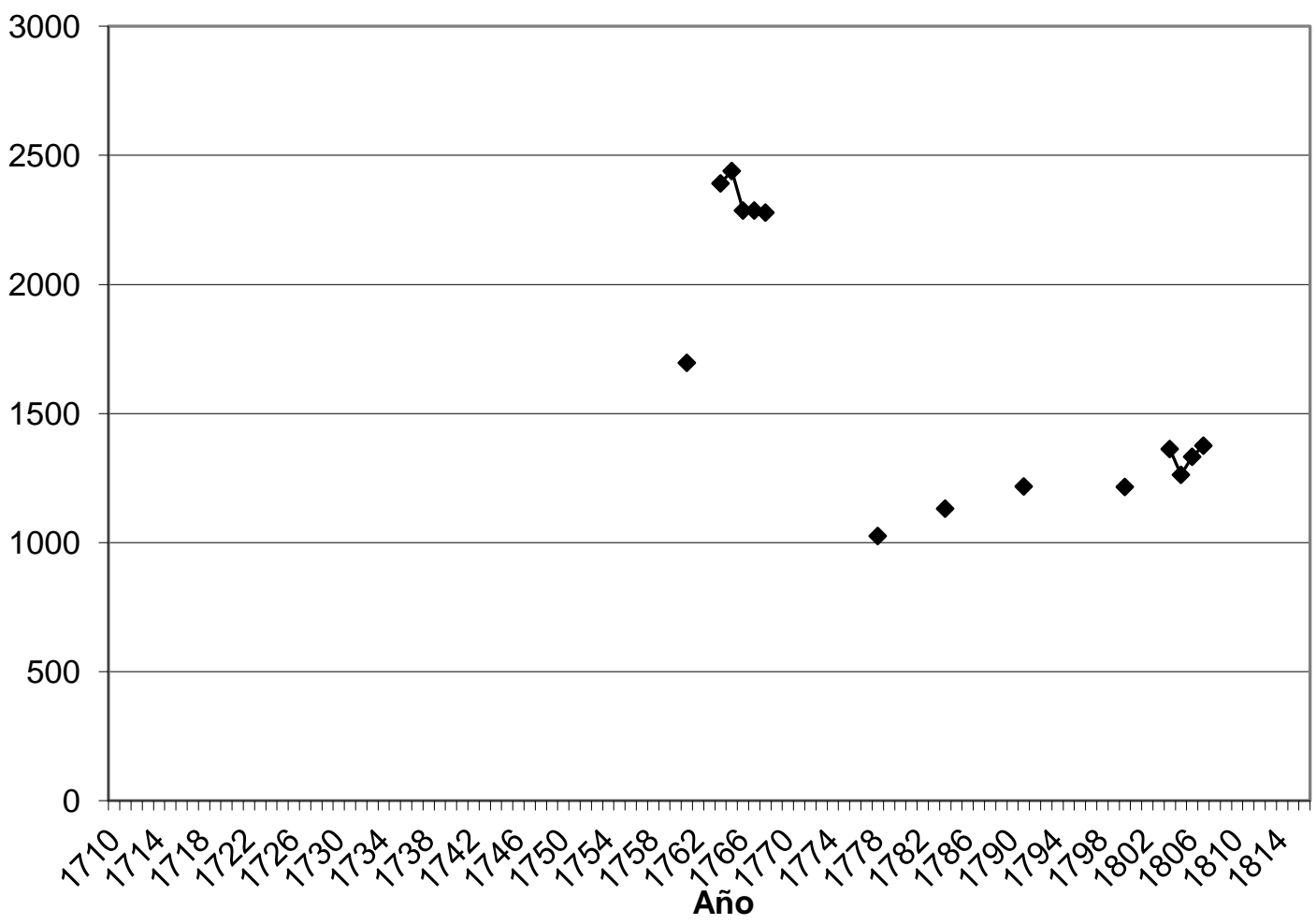

\section{Referencias}

Jackson, R. H. (2004). "Demographic Patterns in the Jesuit Missions of the Rio de la Plata Region: The Case of Corpus Christi Mission, 1622-1802". Colonial Latin American Historical Review v. 13, pp. 337-366.

(2005). "Demographic Patterns on the Chiquitos Missions of Eastern Bolivia, 1691-1767," Bolivian Studies Journal, v. 12, pp. 220-248. 
(2007). "The Post-Jesuit Expulsion Population of the Paraguay Missions, 1768-1803," Colonial Latin American Historical Review, v. 16, pp. 429-458.

(2008). "The Population and Vital Rates of the Jesuit Missions of Paraguay 1700-1767," Journal of Interdisciplinary History 28:3, pp. 401-431.

(2014). "Comprendiendo los efectos de las enfermedades del Viejo Mundo en los nativos americanos: la viruela en las Misiones Jesuíticas de Paraguay," IHS Antiguos Jesuitas en Iberoamerica. V. 2, pp. 88-133.

(2015A). Demographic Change and Ethnic Survival Among The Sedentary Populations On The Jesuit Mission Frontiers of Spanish South America, 16091803: The Formation and Persistence of Mission Communities in a Comparative Context. Leiden: Brill Academic Publishers, 2015.

(2015B). "La población de la Misión de Santa Rosa de Lima (Paraguay)," IHS Antiguos Jesuitas en Iberoamerica, v. 3, pp. 96-110.

Livi-Bacci, Massimo, e Ernesto J. Maeder (2004). "The Missions of Paraguay: the demography of an Experiment." Journal of Interdisciplinary History v. 35, pp. 185-224.

Maeder, E. J., y Bolsi, A. (1976). Evolución y características de la población guaraní de las misiones jesuíticas (1671-1767). Historiografia, v. 2, pp. 113-121.

Maeder, E., y Bolsi, A. (1978). "La población de las misiones de indios chiquitos entre 1735-1766." Folia Histórica del Nordeste, v. 3, pp. 11-26.

Maeder, E. J., y Bolsi, A. S. (1980). La población guaraní de las misiones jesuíticas: evolución y características, 1671-1767 (No. 4). Instituto de Investigaciones Geohistóricas, CONICET, FUNDANORD.

Maeder, E. J., y Bolsi, A. (1982). La población guaraní de la provincia de Misiones en la época post-jesuítica (1768-1809). Folia Histórica del Nordeste, v. 5, pp. 61106. 